\title{
A Commonsense Resolution to the Issue of Determinism versus Rational Agency (aka, free-will)
}

\author{
Larry D. Reid' (iD \\ Professor of Psychology and Neuroscience, The Department on Cognitive Science, Rensselaer Polytechnic Institute, Troy, New \\ York, United States of America
}

$\triangle$ Corresponding Author: Larry D. Reid, E-mail: reidl@RPI.edu

\begin{tabular}{|c|c|}
\hline ARTICLE INFORMATION & ABSTRACT \\
\hline Received: December 08, 2020 & \multirow{7}{*}{$\begin{array}{l}\text { This brief is an essay providing newer perspectives than the traditional (often } \\
\text { religiously oriented) and modern scholarship on free-will versus determinism. The } \\
\text { essay's scope is confined to biological life on Earth and does not address how the } \\
\text { chaos and randomness of the universe had come to have organized planets and suns. } \\
\text { Among the issues raised is that willfulness is not free, rather a cost. The assertion is } \\
\text { made that the agency allowing humanity to make changes deviating from what the } \\
\text { past had wrought is the evolved linguistic skills to communicate first with words, then } \\
\text { signs, then with sentences and then exchanging information in paragraphs allowing } \\
\text { discussions about how to modify the way being lived which, in turn, led to actions and } \\
\text { feedback on the value of their actions, hence progress. }\end{array}$} \\
\hline Accepted: February 16, 2021 & \\
\hline Volume: 3 & \\
\hline Issue: 2 & \\
\hline DOI: 10.32996/jhsss.2021.3.2.4 & \\
\hline KEYWORDS & \\
\hline linguistic skills, rational & \\
\hline
\end{tabular}

discussions

\section{Introduction}

Some insight on the issues of determinism vs free-will can be garnered from a cursory study of humans' abilities and limitations as well as consideration of our evolutionary "family-tree." I explore whether humans have a special feature called free-will, an ambiguous designation carried over from previous centuries' discussions on "Determinism versus Free-will."

\section{A few reasons many can conclude our behaviors have been fully determined.}

We did not choose our parents (our conception) or control what happened during gestation and are generally helpless during our early years. Yet, as we mature, we seemingly, almost universally, wish for and believe that we have, at least, some control of our future. Nevertheless, a goodly number of scholars have concluded our wishes for agency to control the future must be mere fantasy and contrary to hard facts such as those listed.

With the elucidation of the details of genetic code (deoxyribonucleic acid, carrier of genetic information) that was thought to be a sure sign that genetic influence was a major factor in determining all we do (evidence of determinism being understood). Continued study, however, indicates that heredity is not destiny. Further, what an individual might do in changing circumstances has been shown to modify genetic activity sufficiently to consider that genetic activities, obviously necessary for life, are not static. Basically, what are called transcription factors regulate the activity of genes. Here is a quote from Robert Sapolsky's book Behave: "What regulates transcription factors? The answer devastates the concept of genetic determinism: the environment" (Sapolsky, 2017, p 226). In another place, he states: "genes don't make sense outside the context of environment" ( $p$ 227). In the context of this article, there has been considerable writing by scholars that heredity was a major determining factor supporting their line of thinking. Hence, one more reason that all was determined. Despite the revelations of recent science, ideas of genetic determinism are extant.

There is the tyranny of necessity. For species to sustain themselves, they must live sufficiently long to reproduce. That, of course, goes for human beings as well.

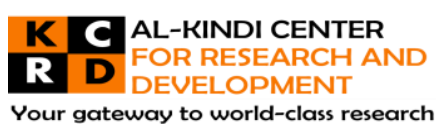

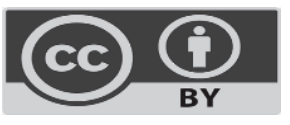

Published by Al-Kindi Center for Research and Development. Copyright (c) the author(s). This open access article is distributed under a Creative Commons Attribution (CC-BY) 4.0 license 
Each person must engage actions that nurture and protect themselves. A highly compelling example: each of us, from birth, on a moment-to-moment schedule, must breathe or die. Breathing is only one of necessary physiological activities.

All must use what is in place that can nurture (e.g., clean air) or approach what is nurturing. At the same time, all must either live in a usually safe environment or must have a well-developed system allowing the avoidance of what can be seriously harmful. Hence the obvious, there must be systems for doing so. Generally, with respect to various species of animals living today, we label these adaptive systems instincts.

Yes, and for sure, satisfying the necessaries does determine much, leaving little room for something like free-will. We either do what is necessary to live or die, and that surely limits what we can do.

We have no memory of the first years of learning to walk and talk. We, therefore, have little personal appreciation of the difficulty of learning such "simple" acts as acquiring the ability to walk upright and gesture for water. It can, therefore, seem to be determined by some agency beyond our selves to have spoken sentences flow without any awareness of how that came to be (to be blessed is not an explanation as good as the explanation that practice tends to prefect what we do). There is apparent magic in the development of a baby into a full professor of philosophy, a rare event, hence, some believe, such must have been preordained by deterministic (magical) forces beyond the ordinary.

Most of daily human behavior is a product of well-established habits. William James (1890) posited that most behavior was habitdriven and then qualified that by suggesting almost all behavior was habit-driven. Further, habits are only loosely controlled by rational thought, and are executed by almost instinct-like surety. This is clearly manifest in the adult behavior by the habits of walking and talking at the same time. These daily activities are so habitual that we have little awareness of the mechanics of walking and speaking while focusing on the content of a conversation as we walk and even walk on uneven ground and around barriers. Our moment-to-moment well-engrained habits involve little conscious awareness and can be taken as a sign that some outside agency has determined our future (notice basketball players who make the sign of the cross before shooting freethrows). The lack of awareness of the details of our executed habits makes it easy to conclude that our actions are determined by forces other than our own will.

The concept that human behavior is determined by the past is fostered by modern psychology and allied disciplines. Outcomes of systematic research fosters the idea that both our evolution and our individual behavior are mostly determined by what was extant previously.

There is the tyranny of the schedule of positive affective events along with the schedule of aversive affective events. We have labels for experiencing positive affective such as being pleased (having pleasure), being satisfied, being comfortable, being happy, etc. And we have labels for the circumstance inducing those affects such as dispensing rewards, positive reinforcement, giving pleasure, and satisfying needs and wishes. We have labels for aversive affective events, paramount among them: experiencing pain, disease, disgust, loss of opportunity for positive affective events and being deprived of different kinds of social support. What causes aversive experiences are such as blows to the body, extreme cold and heat, and other insults perceived by the individual. However, some adversities do not have clear signals such as becoming sick due to pathological infections and hard to detail disturbances in homeostasis. Prior to the discovery of microbes (a relatively recent event, given the prolonged history of humanity) and learning they might cause disease and death, it must have been seen that the occurrences of the aversity and misery of disease had a supernatural cause, some God-like force determining our fate and beyond our personal control.

B.F. Skinner, along with many colleagues, demonstrated over and over that the scheduling of positive reinforcement or relief from adversity (negative reinforcement) exerted strong control of behavior of among rats, monkeys, pigeons, and humans. The demonstrations of the power of various schedules of positive, negative reinforcements and punishments led Skinner to conclude, in his remarkable book Beyond Freedom and Dignity (Skinner, 2002), that behavior was determined by happenings with affective consequences to the extent that there was little left for something akin to free-will. A little recognized feature of the synthesis of the branch of psychology founded by Skinner and colleagues is that by discussions about what was beneficial and fostering of happiness and well-being and how to arrange the schedule of reinforcements to achieve such as a better life and to alleviate misery, was an agency for changing what the past had given. As we will expand on later, knowledge of how best to schedule positive and negative reinforcements to achieve a goal can be an agency for determining some measure of freedom, and, perhaps, dignity and was a demonstration of how to have something other than what had been given by the past.

Of course, and surely, knowledge of how to schedule affective events most effectively can be used to exert remarkable control over those who are subject to the programmers of affective events. There are those who introduce known addictive drugs (pleasure-producing-doses of addictive drugs such as heroin, cocaine, nicotine, and ethanol) to a population can extract vast sums of money from their "subjects" (like experimental subjects in a Skinner box and like those who are in the service of autocrats). Also, a clear example of effective schedules of reinforcement is commercial gambling. All slot machines are 
programmable and are programmed from analyses of constant data-collection on how to deliver money (getting money, for obvious reasons, is nearly a universal reward) for an activity in such a way as to extract more money than was given. Yes, the controlling situation is not much different than the experiment in which rats were trained to work more and more vigorously for food that provided less calories than burned by working for the small amounts of food delivered as an immediate positive reinforcer.

Our dreams, hallucinations, delusions, and imagination together with studied writing by authority figures seem to command the idea that our behavior could easily be determined by outside agencies beyond any ordinary feature of our daily thinking or activities. We dream and often we even remember what we dream. We imagine and even easily imagine super-powers (witness concepts such as angels and characters in Marvel movies). These very personal experiences provide the fodder for concluding that there are agencies outside of our living space, and even outside of our planet, which can with force and ease determine our behavior. However, to preface our main argument, the very activities of impactful dreams and having good imaginations also allow humans to conceive something different than what has been wrought by the past. Hence supporting the rational hope that we can manage something different than what seems to be determined by the past.

There are sociological forces that are surely controlling. For example, some with overly developed egos might conclude that they are blessed by an all-powerful God, hence are unique. Or, they so admire their own actions and thinking, and along with some like-brethren, as to conclude they are the chosen people. Of course, such ideas (so contrary to a full inspection of history which is characterized by the rise and fall of many once successful civilizations) often give rise to bias and prejudice against those supposedly not blessed, not the chosen ones. This idea of being the chosen one or chosen people, by its very nature, suggests that a lot of what most of us do is determined by others with special interactions with powerful forces. Yes, there are autocratic forces that limit our freedom and often control our thoughts.

In discussions of free will vs determinism, there were and are plenty of reasons based in many observations (some examples given above) that the past, writ large, or the forces of nature (as well as imagined supranatural forces) are so pervasive determinates of behavior to conclude that all thoughts and behaviors are determined by the past. To conclude that there is an alternative to the conclusion that all human-behavior is fully determined, one must identify an agency, any agency, that can bend (change) what is apparently ordained. From a commonsense view, I identify an agency that has developed allowing humanity to have some control of the future. That agency is in plain sight and readily observable.

There must be some agency transcending the ordinary biological world for something like free-will to emerge. For many, the agency that can change the future is conceived to be all powerful beings (human-like Gods with extraordinary powers) or some other mysterious force which we might only barely appreciate. Here, we take a more down-to-earth, common sensical, position explaining humans' apparent ability to overcome what the past has given. However, first we need to address the definition of free-will.

\section{There are problems with the concept of free-will.}

Willfulness is not free. It is an effort, a cost. Free seemingly implies no cost, no boundaries, no discussions, something occurring without effort. Having agency to significantly modify what was ordinarily ordained by the past cannot be done by a baby or even a child. It is only when that infant is taught by parenting to cooperate with others that begins the agency allowing for change from previous circumstances. It is the culture of people exchanging information that produces the agency of a single person to have unique ideas; however, if those unique ideas do not stir others to listen then there will be no modification of the past worthy of notice. This does not deny that when we think and act, we perceive we own the act, but owning a thought, by itself does not bend the past toward a meaningful improvement in humans' daily existence.

Reality usually imposes strong constrains on our thinking and actions. However, those having episodes of schizophrenia or extreme narcissism, have perceptions not concordant with reality and one might conclude that such, therefore, is free thought stemming from a lack of the constraints and guidance of reality. Are the hallucinations and delusions of schizophrenia what is meant by the concept of free-will? If you hold that free-will is a condition of not being constrained by realities then, O.K., freewill is exemplified by a schizophrenic episode.

My idea of "free-will" is something different; I abandon the idea that willfulness is free. I find it highly unlikely that any individual can modify what the past has wrought without the aid of others. For example, modern humans have created spaces that are rain-free, snow-free, having a nice comfortable temperature and humidity and walls allowing viewing an outside world. That space also has a supply of clean water, refrigerated space to protect food from spoiling and convenient ways of disposing of waste-products. Other species do have the agency to build nests, shelters, anthills, and honeycombs with some consistency, but they do not write and draw sufficiently to communicate and motivate the activities to build a more modern home. Just as it is seemingly impossible for a single bee or an ant to develop an anthill or a honeycomb, it is impossible for an alone human to build a modern home. That tells us something: our human agency to bend what the past has wrought is not a property of a single 
being, but the work of a group. An extreme example of utilizing the forces of nature (not really changing the forces of nature), consider the development of atomic energy. Our collective knowledge and the work of many created and managed a feature like what we might believe is happening in the sun.

Our uniquely human achievements happen by design that comes from the collective wisdom and actions of groups of people. It took a long time in the history of humanity to have the knowledge to even imagine a modern home. The nice home evolved; it did not just happen by random acts of "free-will" and it is doubtful that a human living thousands of years ago could have even fantasied what it would be like to own a modern family home. The ability to overcome what was thought to be impossible is a unique, emergent talent of human beings: It is not free, but a costly emergent agency centered about the ability to discuss ideas that, in turn, allows humans to not be determined always by the past, i.e., not continually living in caves generation after generation.

\section{A brain prepared to learn language.}

About instincts, we can infer, but cannot detail, what must be the case. During development, the brains of animals are "wired" to execute the essence of the instinct in question. From birth on, all animals must have inherently "wired" neuronal systems executing vital functions such as breathing, moving food through a gut, regulating blood flow in such a manner as to nourish cells of the body and reflexes that prevent choking etc. Brains become organized via development and if they do not become organized the individual will not survive. Brains become organized to do things. Among the things many animals have achieved is the ability to vocalize. Linguistic skills of many animals are merely yelling of pain, warnings of danger, or useful in mating. So, our most primitive humanoid ancestors more than likely had vocalization but little of which is language. Evolutionary changes are small changes not dramatic ones. A brain organized for vocalization could conceivably change just enough for the individuals with that change to have a brain prepared for language. However, being prepared for language is not the same as being born with language ready to emerge in full bloom. Linguistic skills evolve at both the person-level and the cultural-level.

\section{Some comments on the development of the ability to discuss ideas.}

One may ask: If human beings are the only animals on Earth with advanced linguistic skill, then, perhaps, that presents a bit of a problem needing to be discussed (yes, a discussion about discussion). The issue is how could our truly advanced linguistic skills have come into being other than having been ordained by some supra natural force (the idea of a force being supra natural is sort of weird; consequently, I will present ideas about how human's linguistic skills developed)?

The concept of survival of the fittest is a concept that can expand on mere randomness being a step toward agency. Although the concept of the survival of the fittest is usually applied to biological life, it is a relevant concept regarding physical outcomes as well. An example: the power of "survival of the fittest" is surely demonstrable by computer-programs that use "evolution" as a solution to a problem: the programmers merely specify the goal of the "fittest," and then allow an exceptionally large number of progressive trials attempting to reach the specified goal. It can happen, the goal is often achieved. It can happen without the programmers becoming aware of the eventual programming, hence the moniker "black box solution." But, of course, the programmers' goal is determined, hence is not a full account for what is often specified as free-will. In consideration of biological evolution, the goal is freedom from harm and misery in the short run and survival of the species in the long run (not mutually exclusive).

In consideration of the brut forces of natural events relevant to life on Earth, those brut forces can and do select for biological survival. The environment of the planet changes, e.g., whole hemispheres and continents have moved and in so doing made profound changes in local environments. Ecological niches can be optimal for survival of a species until the environment's niche changes (via both physical and biological change, e.g., predation or infectious diseases) that change might hinder reproduction of a species. The very idea of survival of the fittest is dependent of whether a species can survive inevitable changes. Fittest is not a fully adequate characterization, there were very fit dinosaurs when a large asteroid hit the Earth.

Biological processes are not static, "reproduction" is not a perfect process making identical copies of parental individuals. Those that survive gestation and maturation long enough to be a parent are a match between the variability of the biological processes matching the changes in the environment. The idea that our ability to have language, whereas other species do not, is not the outcome of a plan but a series of adaptations which may have been almost superfluous to our species when in one environment but valuable in another. This is what Darwin and Wallace taught us based on their careful observations of nature. However, there is the other feature of the human story. We routinely use language.

The explanation for humans to have the ability to be prepared to have language is likely to be only a small change in the anatomy of the brain of our humanoid ancestors allowing the practice of linguistic skills, hence enhancing linguistic skills that were useful enough for people to use them repeatedly. The human brain was prepared to learn language (being prepared to learn is not an exclusive feature of humans, many animals seem prepared to learn adaptive behaviors germane to their survival). I posit that the earliest and most primitive use of language was found to be useful and satisfying so across generations ancestors eventually developed the skill to speak paragraphs that fellow-humans understood. Yes, we have learned that brains are 
modified by what we do; so that we can do better what we do. Brain plasticity is a characteristic of many mammals, they do learn to do better what they do regularly.

Brain plasticity (a feature of many current mammals) was probably extant among human beings when there was only rudimentary ability to vocally communicate. When primitive linguistic skill became useful and hence practiced more, it literally shaped the brain to do better what was done more routinely. Generation after generation adults talked to their children and their colleagues, hence linguistic skills were improved. Language became a staple of humanity. Our language skills were not planned, ordained, or inherited in full bloom, but nurtured by human interactions across very many generations. They were developed because they served our ancestors' survival in many ecological niches. Language when used routinely becomes so habitual, so instinct-like that it seems to be inherent not developed. The concept of brain plasticity allows the culture of speakers to become experts at linguistic skills.

In accordance with the idea that what animals (of course including us) do can change our brains for doing what we do better (i.e., the idea of brain plasticity known by observation and confirmed by inspection of the anatomy and physiology of the brain), these same ideas are applicable to the issue of Skinner's conclusion that affective consequences are deterministic enough to blunt the possibility of free-will. I said this: "If what we do modifies what we are, then that opens the possibility to use behavioral technology to become what we wish to be" (front pages of the book Sleep, Drugs \& Alzheimer's Disease)( Reid \& Lavash, 2018). That is, if we can discuss what we wish to be, then we have the agency to behave in ways to accomplish what we wish (plan). This surely is not free-will as conceived by some, but such allows humanity to not be totally determined by the past. To broaden the idea even more, if our discussions are not adequate to adaptively engage behaviors in the face of changes in the environment (e.g., a modern threat, the rise of potentially uncontrollable lethal viral infections) we are likely to become an extinct species. On the hopeful side of the issue, our discussions and experiments have led to vaccinations for potentially lethal infections and discussions continue and they are often even rational sometimes.

Yes, it took considerable time, across generations, to develop the ability to construct paragraphs of information understandable by others. We can conclude that the tyranny of necessity combined with the limits of cognition surely limited the agency of our early ancestors. And yes, it follows that now that we have such as the Internet, Wikipedia, and highly sophisticated educational institutions, we now have more agency to change what was problematic in the past into new opportunities.

With our limited agency being dependent on understanding each other's complex ideas, we can strive to something like what is imagined to be heavenly characterized by niceness, gentleness, sensuousness, being comfortable, and being free of disease throughout most of a lifetime. We can by our collective agencies shape our behavior hence the physiologies and anatomy of our brains, in loops of acts, then feedback, to make changes that seemed impossible in the past. We are, in fact, doing just that, but possibly not having sufficient exchange of information to do that well.

\section{Summary}

The idea of free-will has focused on characteristics of an individual human. It is more fruitful to consider what is conceived to be free-will as something different, that is, not focused on an individual but on the ability of a culture to have citizens that can speak full paragraphs clearly understandable by many other citizens. Such an approach is sensible, even common sense, way of appreciating our ability to bend what the past has given.

Human beings are instinct-forming individuals (e.g., the ability to speak sentences) and instinct-overcoming individuals (e.g., engage acts highly unlikely given past events). We have that capacity often throughout a lifetime, allowing for remarkable agency to shape the future. Stated differently, "free-will" is best embodied in a culture allowing freedom of ideas, one encouraging education and the unconstrained flow of ideas. Stated differently, a culture supporting libraries and universities fostering free speech and free flow of ideas is a reasonable circumstance for sustaining what might be called "free-will." And, of course, there is the development of the sciences allowing feedback on the utility of ideas and actions and often creating new insights.

In brief, humans have considerable agency to determine future events as embodied in groups' ability to transmit information to one another made extraordinarily more efficient by reading and writing understandable paragraphs.

My conceptualization of free-will is concordant with the bulk of our experiences, hence is concordant with common sense, common knowledge, and is a solution to the age-old issue of free-will vs determinism which was shaped by theological arguments and often mischaracterized by the individualistic, atomistic, traditional notion of free-will. Yes, there is no, or infinitely small, chance of there being free-will, if free-will is conceived to be a totally free expenditure of energy and distinct from ordinary thinking and acting. However, thought embodied in the exchange of words and drawings, a unique development of humans, is surely an agency that when exercised bends what is supposedly a fixed path. 
The creativity to apparently turn "idyll thoughts" into actions supported by the culture and having obvious value as recognized by fellows can have far reaching consequences. An example of that is the greatness of vaccinations that stopped the progression of some diseases that could not be stopped before, i.e., human agency bending the past to new opportunities, and that is an example of human agency at its best -- or if you wish human willfulness.

If past fully determines future, then the past would surely prevent leaps of cultural achievement. The immutable constraints of the physical forces of the universe would surely curtail creativity, except for that one exception, the emergent ability of humans to process information and transmit it to others. The agency simulating something like free-will is not a potency or capacity of a peculiar human being or an imaginary god, but rather the agency that comes from the long-ago emergence of ability to utter sentences that were understood by their fellows and eventually enhanced writing, reading, and arithmetic of an emergent intellectual culture. Creative actions taken by groups, and their discussion of such, bends the course set by the past and hence are not totally constrained by the past.

The concepts of free-will vs determinism can be foundational ideas for norms of governing. If all is thought to be determined or ordained, then it follows that people who might be evil, handicapped, mentally ill, less competent, or being less than full-fledged humanity can be righteously treated differently than those apparently ordained or blessed or privileged. If, however, human willfulness is a product of using linguistic skills to have discussions, those discussions can have consequences to bend the past toward the better or the worse.

I posit in concert with others, it took many generations of humanoids and human beings to develop the ability to construct paragraphs of information understandable by others. We can conclude that the tyranny of necessity combined with the limits of extant cognitive skills surely limited the agency of our early ancestors. Also, it follows that now that we have such as the Internet, Wikipedia, and highly sophisticated educational institutions, we now have more agency to change what was problematic in the past into new opportunities.

Funding: This research received no external funding.

Conflicts of Interest: The author declares no conflict of interest.

\section{References}

[1] James, W. (1890) The principles of psychology. New York, NY: Henry Holt and Company.

[2] Reid, L. \& Lavash, V. (2018) Sleep, drugs \& Alzheimer's Disease. Brunswick, NY: Hexagon.

[3] Sapolsky, R. (2017) Behave the biology of humans at our best and worst. New York, NY: Penguin Press.

[4] Skinner, B. (1971) Beyond freedom \& dignity. Reprinted 2002, Indianapolis, Indiana: Hackett Publishing Co. 